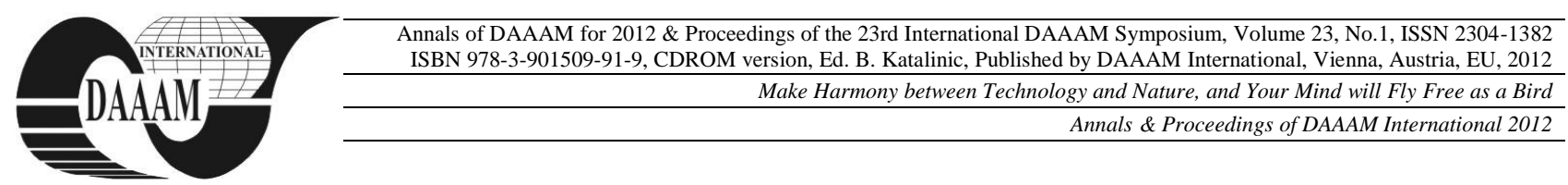

\title{
STATE OF CHARGE ESTIMATES FOR LI-ION BATTERIES USING BQ27541 FUEL GAUGE IN WIRELESS SENSOR NETWORKS
}

\author{
VIERIU, G[eorge] E[mil] \& STAN, A[ndrei]
}

\begin{abstract}
An efficient power management for wireless sensor networks requires precise estimates for the energy reserves available for each node of the network. In case the network uses rechargeable batteries to power the nodes the main characteristic for the energy reserves is the State of Charge (SoC) of the battery. This paper tests four configurations of intelligent Lithium-Ion single cell power modules that integrate the Texas Instruments bq27541 fuel gauge for use in wireless sensor networks (WSNs). To provide a stable output voltage the four tested power modules configuration use linear voltage regulators, a step-up DC-DC converter or a synchronous boost converter. The proposed configurations were tested to determine the precision for state of charge estimates provided by the fuel gauge circuit and also the energy efficiency for each configuration.
\end{abstract}

Keywords: Lithium-Ion, fuel gauge, battery capacity, wireless sensor networks

\section{INTRODUCTION}

Wireless sensor networks (WSNs) are the next step in the technological evolution of measuring devices, providing better performance for complex dynamic applications by using several cheap MEMS (Micro Electro Mechanical Systems). Miniature sensors and other micro-devices are used in the construction of wireless sensor networks nodes to interact with the environment directly. They monitor environmental changes and work together to perform various functions in dynamic and uncertain environments. The usage of sensor networks is the next step in automation in the attempt to simulate the adaptive perception of biological beings. The sensor networks are dynamic and complex systems that combine the understanding of the physical environment with computational and control functions. As a dynamic distributed system such tiny devices must collectively understand the temporal evolution of monitored physical phenomena in order to be able to better predict its effects.

Wireless sensor networks are an elegant solution for a wide range of applications mainly due to the variety of areas in that they may find applicability. Key features of these networks are: the need for self-configuring and self-maintenance, low energy reserves, processing power and memory capacity. Efficient solutions for WSNs applications require an interdisciplinary approach in the design process and also they require rigorous testing.

Wireless sensor networks have specific energy requirements that result from the special operating conditions. Wireless sensor network nodes mostly operate in sleep or hibernate mode with short active periods. Overall the power requirement for the network's nodes is very low and usually a wireless sensor network operates for months or years. These facts pose specific challenges in designing the power packs modules for WSNs.

In this paper the authors tested four power module configurations for WSNs powered by Li-Ion batteries using the bq27541 circuit. Besides calculating the precision for state of charge estimates the author also compared the energy efficiency for the tested configurations. The tested solutions include $2.5 \mathrm{~V}$ and $3.3 \mathrm{~V}$ voltage regulators, a $5 \mathrm{~V}$ step-up DC-DC converter and a $3.3 \mathrm{~V}$ synchronous boost converter. The proposed configurations were tested in low power consumption conditions required for most WSNs.

The development of intelligent power modules for wireless sensor networks that provide accurate data opens up new opportunities for researchers and designers alike. Already proposed routing protocols [1] or dynamic security algorithms [2] can integrate the state of charge predictions, estimate the remaining time to function for each node and determine the optimum solution for efficiently use the energy reserves of the network.

Lithium-Ion batteries are today the favorite power source for most mobile devices (like phones, laptops, PDAs and so on). The widespread use of these batteries has led to the emergence of specialized monitoring circuits. One of these fuel gauge circuits for Li-Ion batteries is the bq27541 from Texas Instruments. The bq27541 fuel gauge uses Texas Instruments patented Impedance Track technology that boasts a 99\% accuracy for battery estimates like remaining capacity and state of charge. The Impedance Track ${ }^{\mathrm{TM}}$ algorithm developed and licensed by Texas Instruments uses measurable values, characteristics and properties of lithium batteries in order to make predictions like the remaining battery capacity for a wide range of battery types. The circuit monitors the battery status in both charging and discharging cycles using a sense resistor with value between 5 and $20 \mathrm{~m} \Omega$.

In [3] the authors of the application report proved that the accuracy of the Impedance Track ${ }^{\mathrm{TM}}$ algorithm is over $99 \%$ for phone applications. The fuel gauge used is bq27500, an older version of the bq27541 we used in our tests. The purpose our paper is to determine the optimal power module solution for WSNs using a single Li-Ion battery (monitored by a bq27541 fuel gauge) that provides constant output voltage. The criteria used in choosing the optimal solution between the tested configurations are state of charge (SoC) estimation accuracy and overall energy efficiency. 


\section{EXPERIMENTAL SETUP}

The proposed power pack configurations use an 18650 rechargeable Li Ion battery that is monitored by a bq27541 v2.0 fuel gauge circuit. The 18650 cell type is commonly used in laptop batteries, in LED flashlights and even cars (Tesla Roadster, a sports car produced by the electric car firm Tesla Motors in California). The LiIon cell is produced by Trustfire and it is rated at $2500 \mathrm{mAh}$ capacity. This cell and 2 others were reviewed in [4]. The battery provides a voltage between $4.2 \mathrm{~V}$ and $2.75 \mathrm{~V}$, and has a protection circuit that disconnects the cell if the voltage crosses $4.2 \mathrm{~V}$ while it is charging (overcharge) and drops below $2.75 \mathrm{~V}$ while discharging (cutoff voltage).

In order to provide a stable voltage output for the power pack there were tested four configurations:

- TPS73625: a 2.5V low-dropout (LDO) linear voltage regulator with $75 \mathrm{mV}$ dropout voltage, produced by Texas Instruments

- TPS73633: a 3.3V low-dropout (LDO) linear voltage regulator with $75 \mathrm{mV}$ dropout voltage produced by Texas Instruments

- MAX856: a 5V step-up DC-DC converter, produced by Maxim

- TPS61201: a 3.3V low input voltage synchronous boost converter with automatic transition between Boost Mode and Down Conversion Mode, produced by Texas Instruments.

The battery pack was connected to a simple constant current load that was also used in [4] to test the batteries. The schematic for the load circuit is shown in Fig. 1. The load uses a current sink with a LM324 low power operational amplifier, RFP30N06LE logic level NChannel power MOSFET $\left(30 \mathrm{~A}, 60 \mathrm{~V}, \mathrm{r}_{\mathrm{DS}(\mathrm{ON})}=47 \mathrm{~m} \Omega\right)$, a $1 \Omega, 1 \%$ precision power resistor and a voltage divider for the $5 \mathrm{~V}$ external power supply. Using the $5 \mathrm{k} \Omega, 1 \%, 10$ turns potentiometer (R2) the voltage over R1 resistor can be set between 0 and $2.6 \mathrm{~V}$ and thus the current load can be set anywhere between 0 and $2600 \mathrm{~mA}$.

The Impedance Track algorithm requires calibration of the bq27541 for each battery type to function. The bq27541 circuit calibration process follows the following steps from bqEasy section of the bqEVSW software [5]:

STEP 1: discharge the battery to empty

STEP 2: wait 5 hours for the battery to relax

STEP 3: initialize the Impedance Track Algorithm

STEP 4: charging the battery to full

STEP 5: wait 2 hours for battery to relax

STEP 6: discharge the battery to empty

STEP 7: wait again 5 hours for battery to relax

STEP 8: save the data collected to a Golden Pack file STEP 9: update the Golden Pack Data.

After the calibration process is complete and the data loaded into the bq27541, the circuit can provide data about the battery: temperature $\left({ }^{\circ} \mathrm{C}\right)$, voltage $(\mathrm{mV})$, average current (mA), normal available capacity (mAh),

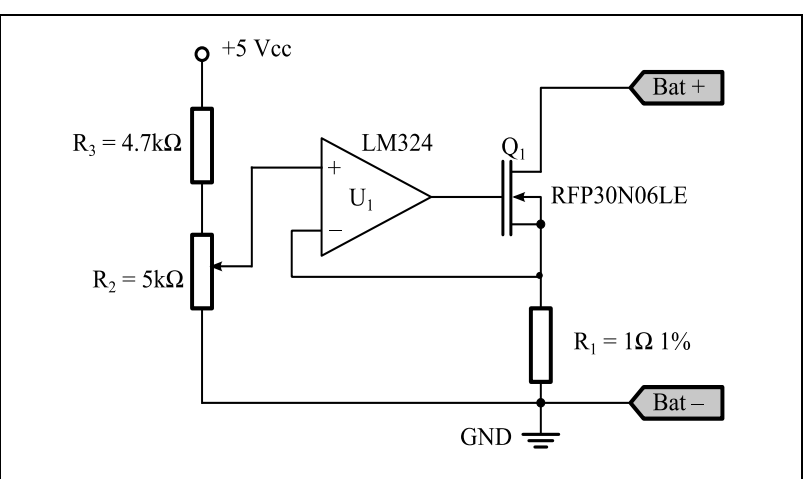

Fig. 1. Schematic for the constant current load

full available capacity (mAh), remaining capacity (mAh), full charge capacity (mAh), available energy (mWh), available power $(\mathrm{mW})$, state of charge $(\%)$ etc.

\section{TEST RESULTS}

For the first test the $2.5 \mathrm{~V}$ LDO voltage regulator was used. The load was set to $100 \mathrm{~mA}$ and a fully charged LIIon battery was monitored while it was discharged trough the regulator connected to the constant current load.

After the discharge was complete the data provided by the fuel gauge and saved by the bqEVSW was compared with the ideal linear discharge graph. The estimation error for the logged data is shown in Fig. 2. There are two means to estimate the state of charge of the battery: one is directly use the state of charge ( $\mathrm{SoC}$ ) value provided by the fuel gauge and the other is to calculate it using the remaining capacity ( $\mathrm{Cap}$ ) and full capacity (FullCap) values. The $S o C$ value is represented as an integer with values between 0 and 100, and thus has lower precision compared with the obtained Cap/FullCap value that is represented as a floating point number. Tab. 1 contains the average and maximum variation error for the two estimation values.

Beside the precision for the state or charge (defined as the remaining battery capacity expressed in percentages) we also monitored the energy efficiency for each power module configuration as the report between the measured energy ( $\mathrm{mWh}$ ) measured at the output of the power module (that provides constant voltage and current) and the measured energy drawn from the battery for a complete discharge cycle.

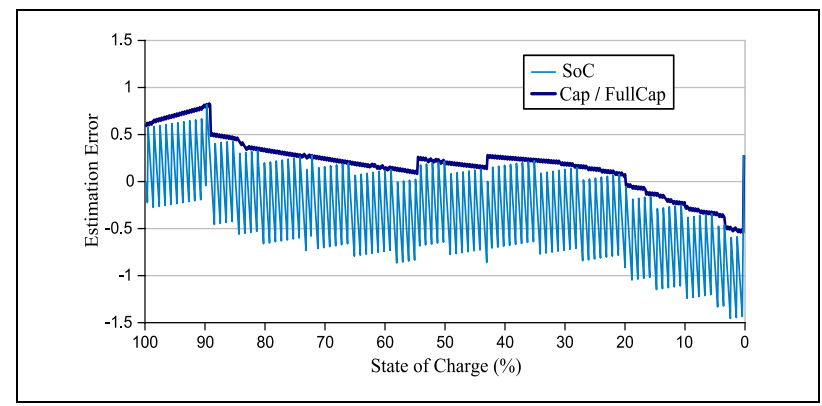

Fig. 2. Estimation error for the 2.5V LDO configuration

\begin{tabular}{|c|c|c|}
\hline Method of estimation & $\begin{array}{c}\text { Average error } \\
\text { value }\end{array}$ & $\begin{array}{c}\text { Maximum error } \\
\text { value }\end{array}$ \\
\hline SoC & 0.3131144063 & 1.4543227038 \\
\hline Cap/FullCap & 0.1861998833 & 0.8304108806 \\
\hline
\end{tabular}

Tab. 1. Estimation error for the 2.5V LDO configuration 


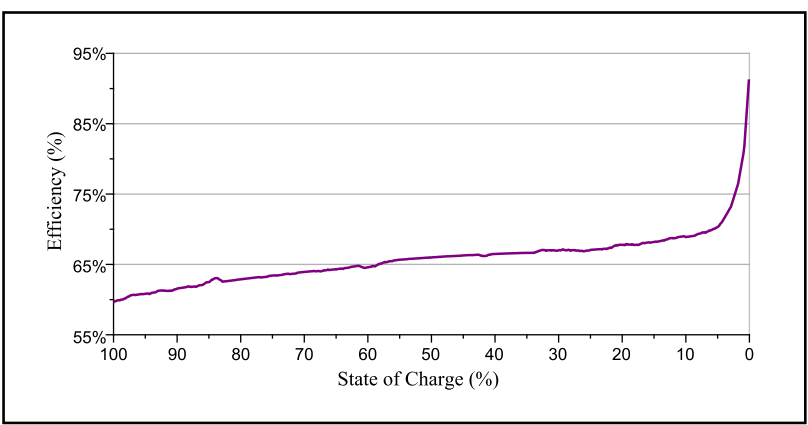

Fig. 3. Energy efficiency for the 2.5V LDO configuration

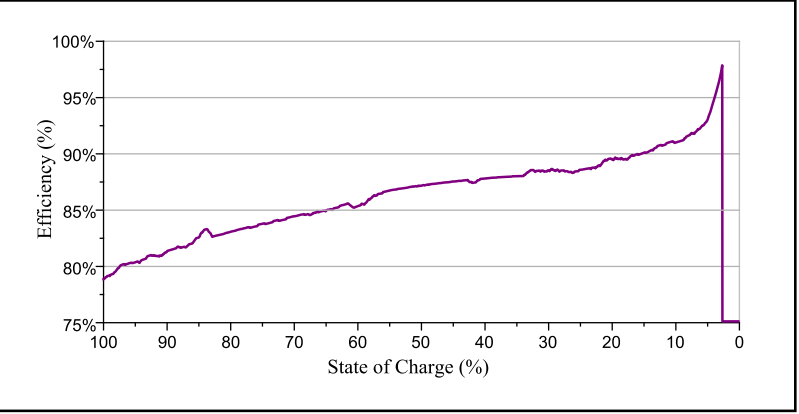

Fig. 4. Energy efficiency for the 3.3V LDO configuration

For the 3.3V LDO configuration the values obtained for the state of charge estimations were very similar to the ones from Fig. 2 and Tab. I and will not be included in the paper. The evolution of the energy efficiency calculated for the two configurations can be seen in Fig. 3 for 2.5V LDO configuration and Fig. 4 for the 3.3V LDO configuration. In Fig. 4 the energy efficiency is considered 0 after the LDO output voltage drops under $3.3 \mathrm{~V}$ (battery voltage output drops below $3.375 \mathrm{~V}$ ). The average energy efficiency was $67.83 \%$ for the $2.5 \mathrm{~V}$ LDO and $86.37 \%$ for the $3.3 \mathrm{~V} \mathrm{LDO}$.

The third test configuration used a 5V MAX856 stepup DC-DC converter and was the only configuration tested that provided $5 \mathrm{~V}$ output voltage. The process vas repeated with the same battery after it was recharged. The data acquired in this configuration is shown in Fig. 5 and Tab. 2.

The last configuration tested used a $3.3 \mathrm{~V}$ fixed voltage TPS61201 synchronous boost converter. Compared to a DC-DC converter the TPS61201 can function in two modes: Boost Mode and a Down Conversion Mode. The circuit switched automatically between these two modes based on the input voltage value. The data obtained can be seen in Fig. 6 and maximum variation and average values in Tab. 3 .

The evolution of the energy efficiency for the last two configurations is shown in Fig. 7 (for the MAX856) and Fig. 8 (for the TPS61201).

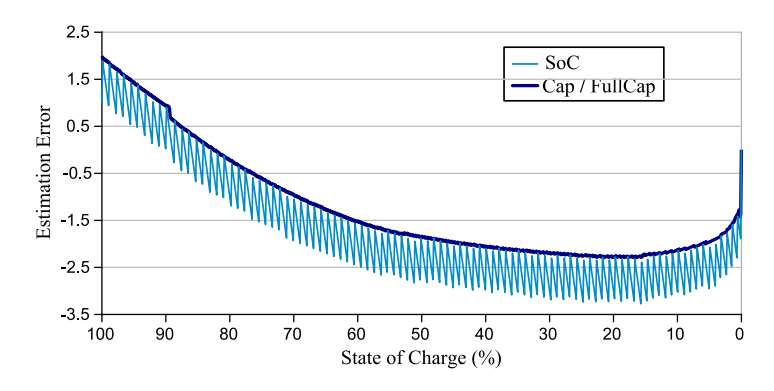

Fig. 5. Estimation error for the 5V Step-Up configuration

\begin{tabular}{|c|c|c|}
\hline $\begin{array}{c}\text { Method of } \\
\text { estimation }\end{array}$ & $\begin{array}{c}\text { Average error } \\
\text { value }\end{array}$ & $\begin{array}{c}\text { Maximum error } \\
\text { value }\end{array}$ \\
\hline SoC & 1.7183514289 & 3.2735076159 \\
\hline Cap/FullCap & 1.2285468588 & 2.2938250444 \\
\hline
\end{tabular}

Tab. 2. Estimation error for the 5V Step-Up configuration

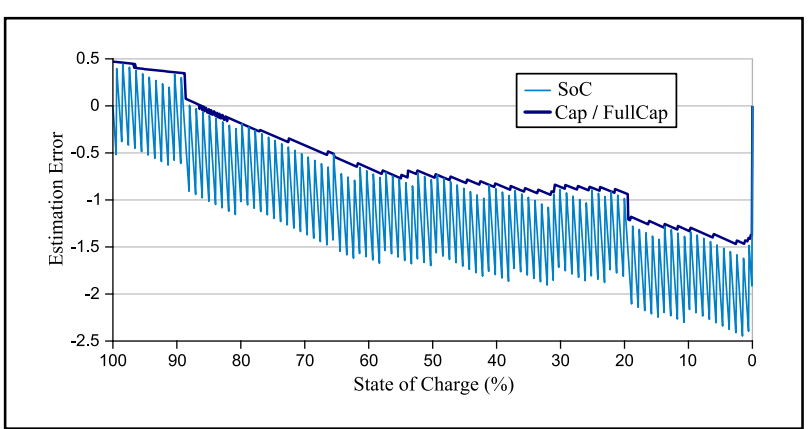

Fig. 6. Estimation error for the 3.3V boost converter configuration

\begin{tabular}{|c|c|c|}
\hline $\begin{array}{c}\text { Method of } \\
\text { estimation }\end{array}$ & $\begin{array}{c}\text { Average error } \\
\text { value }\end{array}$ & $\begin{array}{c}\text { Maximum error } \\
\text { value }\end{array}$ \\
\hline SoC & 1.1325387993 & 2.4475844126 \\
\hline Cap/FullCap & 0.635681968 & 1.4680832324 \\
\hline
\end{tabular}

Tab. 3. Estimation error for the $3.3 \mathrm{~V}$ boost converter configuration

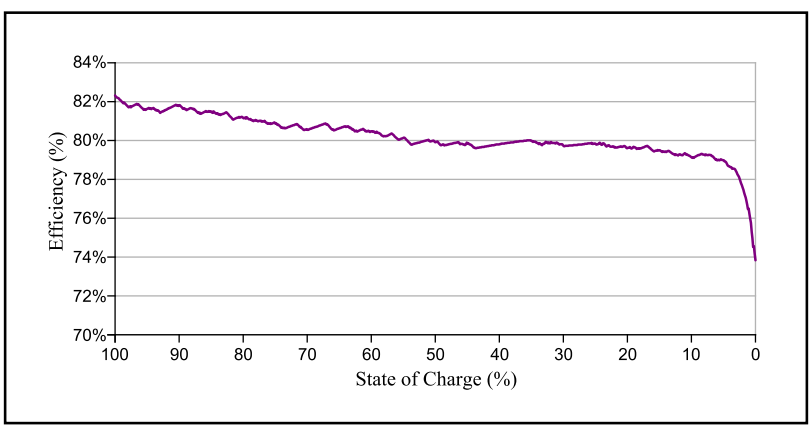

Fig. 7. Energy efficiency for the for the 5V Step-Up configuration

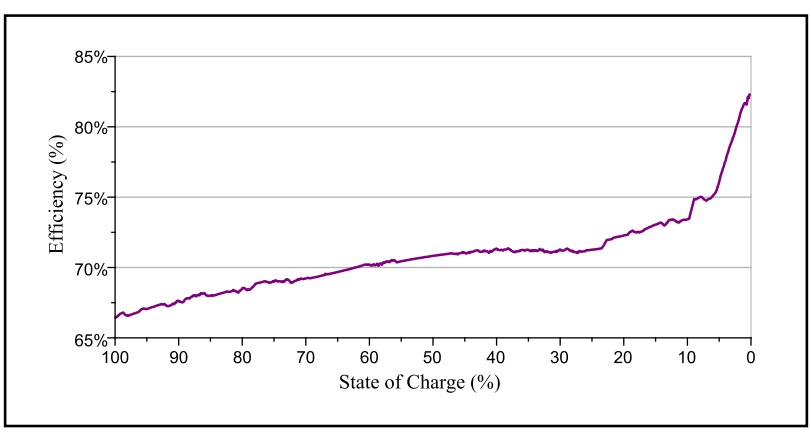

Fig. 8. Energy efficiency for the 3.3V boost converter configuration

Tab. 4 contains the energy efficiency for the four tested configurations for a complete discharge cycle for the same Li-Ion cell. After each test the battery was fully charged and we waited at least 5 hours to start the next test. After the tests were completed we remade the measurements with a different battery of the same type and obtained similar results. Fig. 9 contains the evolution of the energy efficiencies for all configurations.

\begin{tabular}{|c|c|}
\hline Configuration tested & Energy Efficiency \\
\hline TPS73625 (2.5V LDO) & $67.83 \%$ \\
\hline TPS73633 (3.3V LDO) & $86.37 \%$ \\
\hline MAX856 (5V step-up DC-DC) & $80.18 \%$ \\
\hline TPS61201 (3.3V boost converter) & $70.73 \%$ \\
\hline
\end{tabular}

Tab. 4. Energy efficiencies for the four configurations tested 


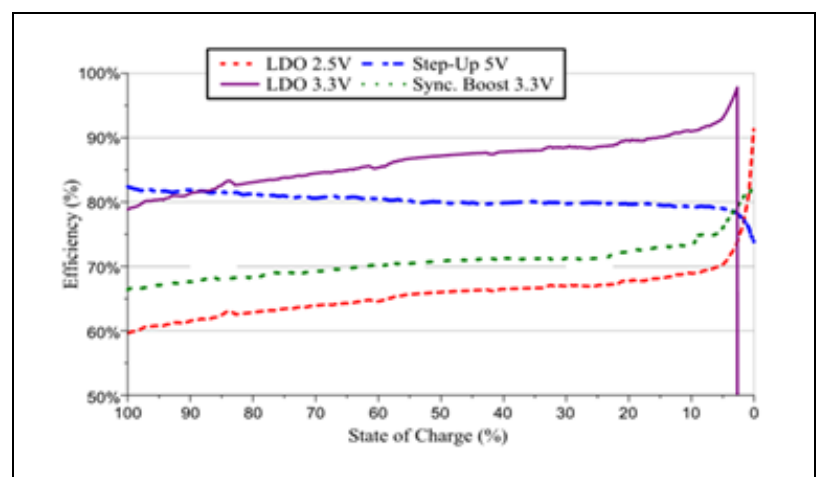

Fig. 9. Energy efficiency for the four tested configurations

The energy efficiency for the $3.3 \mathrm{~V}$ synchronous boost converter was lower than expected; mainly because the circuit was functioning most of the time in step-down mode (according the circuit datasheet the energy efficiency is significantly lower in step down mode). In Fig. 8 we can observe an increase in energy efficiency for the third configuration (TPS61201 3.3V boost converter) when the battery state of charge drops below $10 \%$. This is due to TPS61201 switching to boost mode when the circuit input drops below $3.3 \mathrm{~V}$.

There is a third method to obtain the state of charge value based on the data provided by bq27541 fuel gauge. This method uses NomAvailCap and FullAvailCap value provided by the circuit, but it provided poor results compared with Cap/FullCap combination. Yet for the fourth configuration (TPS61201 boost converter) this method provided a more linear behavior. The estimate provided by NomAvailCap/FullAvailCap can be observed in Fig. 10.

\section{CONCLUSIONS}

All aspects of WSNs operation depend on the amount of available energy for each node of the network. Spending the nodes energy efficiently requires first of all a clearer picture of the energy reserves for the whole network. Of course it has great importance for WSNs designers the degree of trust we can put on these state of charge estimations. This article sought to answer to these matters and in addition for the tested configurations the energy efficiency of the power module (battery, fuel gauge and voltage regulator) was determined.

From the tests results we can see that the precision estimates for state of charge values for devices powered by these power modules are satisfactory with best values (from the four tested configurations) obtained for voltage regulators (average estimated error of $0.18 \%$ and max value of $0.83 \%$ ).

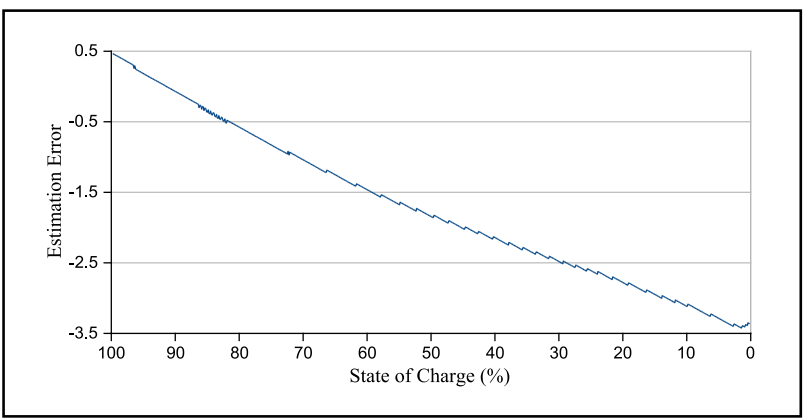

Fig. 10. Estimation error for the 5V Step-Up configuration using NomAvailCap/FullAvailCap values
Because of the low voltages and currents required for the power packs for WSNs we can see that best efficiency is obtained for the simplest circuits (voltage regulators). As proved the $3.3 \mathrm{~V}$ LDO was more efficient than the $3.3 \mathrm{~V}$ boost convertor and also provided better precision for the state of charge predictions. The LDO is also cheaper and doesn't require additional components like inductor and capacitors. The only advantage of the TPS61201 boost converter is that it can be set to automatically disconnect at a set voltage, and thus can be used with unprotected batteries.

In this paper we proved that state of charge predictions for Li-Ion single cell batteries can have very high accuracy despite the nonlinearities introduced by the additional circuits required to regulate the out voltage. There are numerous other circuits that can be used to obtain the constant voltage output. The results of our tests can be compared with other possible configurations.

The data collected in these tests can be used to select the optimal solution for creating intelligent power modules for wireless sensor networks based on single cell Li-Ion batteries.

The state of charge predictions can be used to make estimates about the remaining functioning time for a battery powered device. The accuracy of the remaining functioning time estimates is certainly dependent on the precision of the state of charge estimates. In WSNs a precise estimations of remaining functioning time for each node may prove to be very valuable in choosing the best network configuration in order to prolong the function time of the network as a whole.

In this paper we succeeded in measuring the precision of state of charge estimates for a power module composed of a single Li-Ion cell that use Texas Instrument's bq27541 fuel gauge and four circuits to regulate the output voltage.

There are currently available protected Li-Ion batteries that have attached circuits that provide overcharge and cut-off discharge protection. The emergence of cheap and easy to use fuel gauge circuits for Li-Ion batteries make us hope for their standardization so the battery manufacturers may include such circuits in the actual battery besides the current battery protection.

\section{REFERENCES}

[1] Al-Karaki, J.N.; Kamal, A.E. (2004), Routing Techniques in Wireless Sensor Networks: A Survey, Avaiable from: home.engineering.iastate.edu/ kamal/Docs/kk04.pdf Accessed: 2011-06-20

[2] Botezatu, N.; Manta V.; Vieriu G. (2011), A clustering algorithm for negotiating security in wireless mesh networks, 15th International Conference on System Theory, Control, and Computing (ICSTCC), 14-16 October 2011, Sinaia, Romania pp. 1-6

[3] Application Report (2008), Impedance Track Fuel Gauge Accuracy Test for GSM Phone Applications, Avaiable from: http://www.ti.com/lit/ug/ sluu307a/sluu307a.pdf Accessed: 2012-03-08

[4] Vieriu, G.E. (2012). Lithium-Ion battery test for wireless sensor networks, Buletinul Institutului Politehnic din Iasi, Automatic Control and Computer Engineering Section, tome LVIII, Fasc. 1, "Gheorghe Asachi" Technical University of Iasi, pp. 113-121

[5] User's Guide (2010), bqEASYтм for Single Cell Impedance Track ${ }^{\mathrm{TM}}$ Devices, Avaiable from: http://www.ti.com/lit/ug/ sluu307a/sluu307a.pdf Accessed: 2012-03-08 